(2) Open Access Full Text Article

PERSPECTIVES

\title{
Impact of COVID-19 on Urology Practice in Saudi Arabia
}

This article was published in the following Dove Press journal:

Risk Management and Healthcare Policy

\author{
Meshari A Al-Zahrani (D' \\ Mohammad Alkhamees (D) \\ Sulaiman Almutairi (D) \\ Ahmed Aljuhayman' \\ Sultan Alkhateeb ${ }^{2,3}$ \\ 'Department of Urology, College of \\ Medicine, Majmaah University, Al- \\ Majmaah, Saudi Arabia; ${ }^{2}$ Department of \\ Urology, King Faisal Specialist Hospital \& \\ Research Center, Riyadh, Saudi Arabia; \\ ${ }^{3}$ College of Medicine, Alfaisal University, \\ Riyadh, Saudi Arabia
}

\begin{abstract}
From the moment the World Health Organization (WHO) declared COVID-19 to be a pandemic disease, COVID-19 began to affect the lives of many healthcare providers worldwide. In response to this pandemic, urology departments and training residency programs implemented urgent measures to reduce outpatient clinics, adopted the use of telemedicine, regulated emergency and outpatient urological procedures, promoted the use of operating theatres, and developed the use of sustainable e-learning alternatives to traditional urology educational activities. We reviewed the response of urologists in Saudi Arabia to the COVID-19 pandemic and how they react to the emerging pandemic both for patients and for healthcare of urologist personnel.
\end{abstract}

Keywords: Saudi Arabia, urology, COVID-19, telemedicine, residency

\section{Introduction}

In late December 2019, a novel coronavirus was detected in Wuhan, China, ${ }^{1}$ belonging to the family named SARS-CoV-2 (previously known as 2019-nCoV). It became known as coronavirus disease 2019 (COVID-19), as named by the World Health Organization (WHO) in February 2020. The disease was later declared a pandemic in March 2020. ${ }^{2}$ This disease leads to various degrees of pneumonia, acute respiratory distress syndrome (ARDS), and, in severe cases, to the need for mechanical ventilation support or admission to the intensive care unit (ICU). ${ }^{3,4}$ During the COVID-19 pandemic, global efforts were combined to serve, educate, and prepare healthcare providers (HCPs) to equip and support resources and personnel to face such crisis.

From 30 December 2019 to 11 October 2020, the WHO reported over 37 million cases and 1 million deaths globally. ${ }^{5}$ On 5 October 2020, more than 2.2 million new cases and 39,000 deaths of COVID-19 have been reported across all six WHO regions, which was considered the highest number of reported cases so far in a single week. ${ }^{5}$ Since the outbreak, the number of cases has been continually rising and numerous vaccines have been initiated in many clinical trials. Currently, the WHO is launching the Access to COVID-19 Tools Accelerator, which is working on diagnostics, treatment, vaccines and health system strengthening. It includes COVAX, which is supporting the development of 9 vaccines, with more in the pipeline, and aims to fairly distribute 2 billion vaccine doses by the end of $2021 .^{5}$

In Saudi Arabia, a group from the biomedical engineering faculty from Imam Abdulrahman Bin Faisal University used the autoregressive integrated moving average
Correspondence: Meshari A Al-Zahrani Teaching Assistant, Department of Urology, College of Medicine, Majmaah University, Al-Majmaah, I1952, Saudi Arabia

Tel +00966569990693

Fax +00966164042500

Email ma.alzahrani@mu.edu.sa
Risk Management and Healthcare Policy 2021:14 1379-1392

DovePress in $\square$ 
(ARIMA) model to predict the expected daily number of COVID-19 cases. The results showed that the trend in cases was due to continue growing if strict precautionary steps and control measures were not implemented to limit the spread of infection. ${ }^{6}$ The Saudi government announced its first case of COVID-19 on 2 March 2020 when a patient from outside the country was confirmed as being infected. The infection was detected as a result of the Saudi Ministry of Health $(\mathrm{MOH})$ applying a protocol for detecting the virus across all the country's national borders. ${ }^{7}$ As of 20 October 2020, there were a total of 7,403,020 tests performed since the pandemic started, a total of 342,583 confirmed cases and 8487 were active cases, with 844 cases requiring ICU admittance, and a total of 5201 deaths. Surprisingly, the recovery rate has been high, with a total of 328,895 recovered cases, resulting in a case mortality rate of $2 \%$ and a recovery rate of $98 \%{ }^{8,9}$

As is the case worldwide, this crisis has affected healthcare at the population level in Saudi Arabia. However, following the recommendations and guidelines of the Saudi MOH and the Saudi Center for Disease Prevention and Control (SaudiCDC - Weqaya), ${ }^{10,11}$ the Saudi government implemented scientific measures to control the spread of COVID-19 from the moment the crisis was announced, and internal and external lockdowns in the early stage of the pandemic were successfully implemented.

Saudi MOH/SaudiCDC COVID-19 Coronavirus Disease Guidelines and Protocol for patients suspected/confirmed with COVID-19 $9^{10,11}$ were established since the pandemic crisis evolved and frequently updated, targeting different aspects of core health care system for both HCPs and general public starting from prevention to diagnosis and management. The Saudi $\mathrm{MOH}$ has called on citizens and residents to call the (937) service center in case of any inquiry regarding the COVID-19 24/7, stressing the importance of protection from respiratory diseases in general. ${ }^{12}$ Saudi MOH has devoted (Tetamman) "Rest Assured" Clinics $^{13}$ to serve individuals who show COVID-19 symptoms, such as: fever, coughing, sore throat, shortness of breath, chest pain, diarrhea, or loss of taste and smell. The clinics receive patients at any time, without appointments for Saudi citizens and non-Saudi residents legal/illegal residents or illegal immigrant workers. Awareness platform of Saudi $\mathrm{MOH}^{14}$ provided the COVID19 Prevention Guideline in various languages and distributed on different social media accounts, websites, local broadcast channels and phone short message service (SMS).
Remarkably, beside many other efforts as a result, the spread of infection and the mortality rate were maintained at low rates, which helped decrease the burden on the healthcare system.

In this regard, the main goal of this article is to share our experiences and recommendations, highlighting the impact on the urology service and demonstrating the role of urologists during the COVID-19 outbreak in Saudi Arabia.

\section{Worldwide Impact of COVID-I9 on Urologist}

COVID-19 resulted in substantial global impact on urological care and among urologist. Worldwide, Urology practice has been revolutionized as a result of COVID-19's unprecedented effect on health care system. Urologists in Saudi Arabia, like many other HCPs around the globe, followed this emerging pandemic with interest. A study that included 529 physicians from different regions in Saudi Arabia showed that the COVID-19 pandemic had a negative psychological impact on physicians in Saudi Arabia, with the most commonly reported feelings being worry, isolation, and fear. ${ }^{15}$ A large sample international survey ${ }^{16}$ including a total of 1004 urology professionals from six continents showed $41 \%$ respondants reported that their hospital employees had COVID-19 infection, 33\% of them felt they get adequate personal protective equipment (PPE) and $47 \%$ expressed fear of returning to work. In this study, estimated diminished urological services with COVID-19 outbreak effect remarkably increased and result in a delay of $>8$ weeks in outpatient clinics, outpatient investigations and procedures, and urological surgeries in $28 \%, 30 \%$ and $31 \%$ respectively.

Worldwide, these foreseeable changes will put significant strain on the urological community, primarily on amount of work, surgical patients' inflow, and operation waiting lists. Thus, looking to recent literature, we attempt to explore these changes and the response of urologists in Saudi Arabia to this current pandemic.

\section{Impact of COVID-I 9 on Urology Outpatient Clinics}

Since the outbreak of the COVID-19 pandemic around the globe, the Saudi MOH was quick to implement appropriate action regarding clinical practice within the country. This was in accordance with initial data from WuhanChina, the WHO, and the Center for Disease Control and 
Prevention (CDC), which recommended practice guidelines for physicians to control the COVID-19 infection. ${ }^{17-19}$ These recommendations included the use of PPE such as a filtering facepiece (FFP; FPP2 and FPP3 for aerosol-producing procedures), single-use protective clothing, protective eyewear, frequent and accurate surface disinfections, ventilation of outpatient services, and washing of hands with alcoholic solutions.

Health care policies are continually changed during the COVID-19 pandemic and encouraged access to Telemedicine (TM), "clinical services like telephone or video communication with patient", and Telehealth (TH), "non-clinical services like use of mobile applications and digital health information platforms", and promoted using these developing technology to provide acute, chronic, primary, and specialty care. ${ }^{20}$ Many professional medical societies encouraged TM services and provided guidance for HCPs in this developing field during the pandemic. ${ }^{21,22}$ Recently, a comprehensive review of the recommendations by International and European associations on priority procedures outlined the potential role of TM to optimize resources in the current and future scenarios. ${ }^{23}$ It was remarkably observed in literature the increase in use of
TH and TM among the urological community. ${ }^{24,25}$ One of the advantages of those technologies is that patient health outcomes can be improved. ${ }^{26}$ During the pandemic the TM and smart working platforms (Figure 1) provide specialized clinical support for urologists at a distance using technological tools as a logistically feasible alternative to face-to-face (FTF) consultation. ${ }^{25}$ In a video visit, some patients may face internet connection difficulties due to technical issues, so the best alternative option in such scenario would be a telephone call. ${ }^{25}$

a A recent systemic review, explored the $\mathrm{TH}$ use in urology practice and what has been useful during and after the COVID-19 pandemic. ${ }^{27}$ The authors found the TM has been implemented successfully in selected patients with prostate cancer $(\mathrm{PCa})$, urinary incontinence, pelvic organ prolapse, uncomplicated urolithiasis, and urinary tract infections. The imitation of this review was not explored other malignant urological conditions and other highly prevalent benign conditions, such as benign prostatic hyperplasia (BPH), overactive bladder syndromes, and sexual dysfunction, extensive evaluation are required.

The Saudi $\mathrm{MOH}$ has developed the Digital Health Strategy Update in 2018 and has been created to support

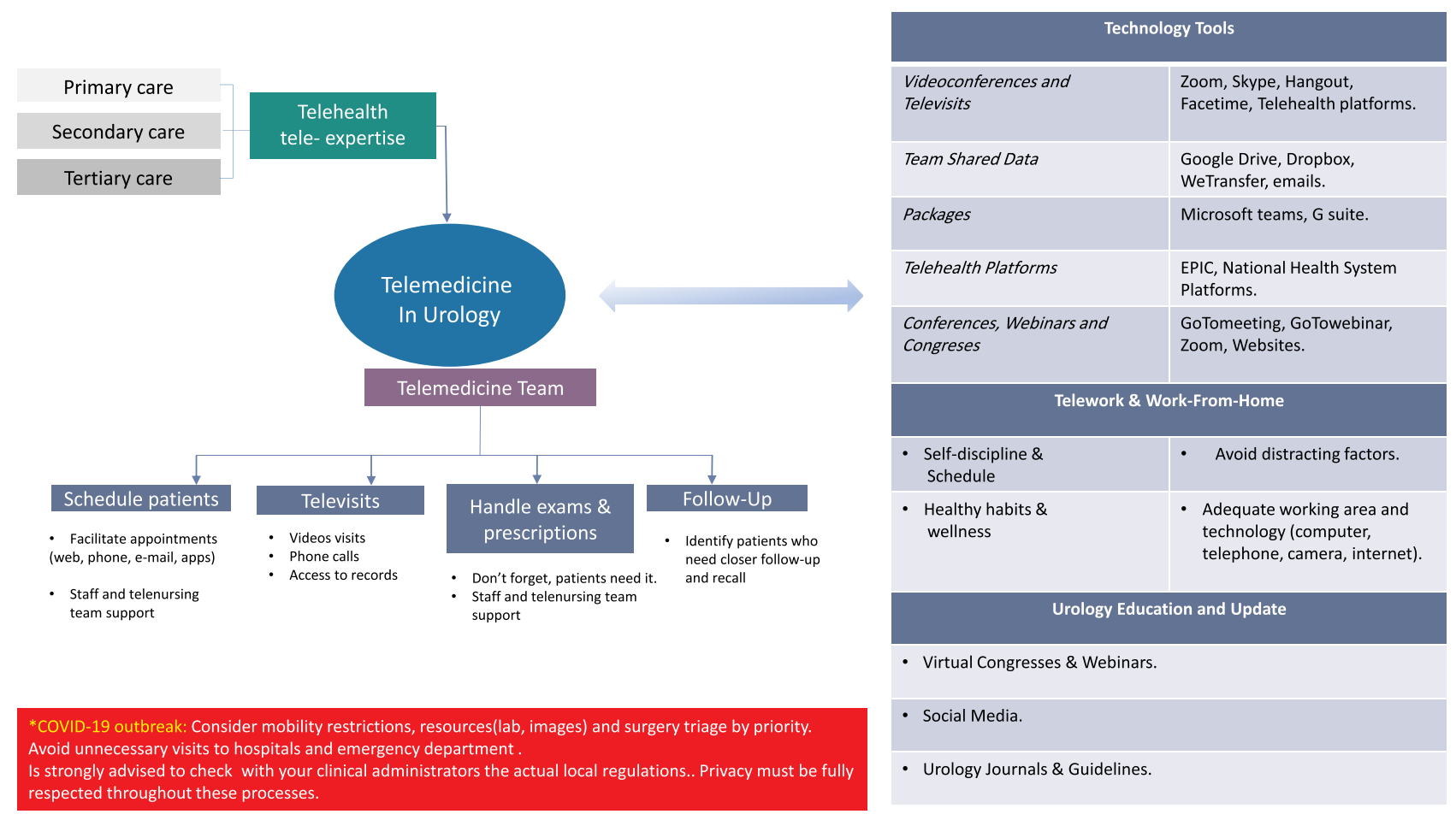

Figure I Telemedicine and telehealth platform applications in urology during COVID-19 pandemic time.

Note: Reprinted from European Urology, 78/6, Moises Rodriguez Socarrás, Stacy Loeb, Jeremy Yuen-Chun Teoh, Maria J. Ribal, Jarka Bloemberg, James Catto, James N’Dow, Hendrik Van Poppel, Juan Gómez Rivas, Telemedicine and Smart Working: Recommendations of the European Association of Urology, 8I2-8I9, Copyright (2020), with permission from Elsevier. ${ }^{25}$ 
delivery and reflect implementation of the Saudi Vision 2030 for the MOH Healthcare sector, facilitate cluster autonomy, the need for digital reinvention and provide rapid digital change. ${ }^{28}$ As a result, $\mathrm{TH}$ and $\mathrm{TM}$ has been one of the essential factors for control of COVID-19 spread and has helped to flatten the growth curve in Saudi Arabia. TH mobile applications (eg, Seha, Mawid, Tawakkalna, Tabaud, and Tetamman) have found effective tools to facilitate delivering healthcare to persons with COVID-19 and tracking of COVID-19 patients. ${ }^{29}$ Before the pandemic, a cross-sectional survey on the knowledge, attitude, and practice of Saudi and non-Saudi parents to TH mobile applications (eg, Seha and Mawid) was recently conducted. The study reported that $86 \%$ of the respondents acknowledged the potential benefits of these mobile applications and had used them before to book appointments for their children. ${ }^{30}$ In another public survey, $51 \%$ of the users were satisfied with the current services of TH mobile applications (eg, Seha and Mawid). ${ }^{31}$ During the pandemic, there were $>2$ million users for the $\mathrm{TH}$ applications established by the Saudi $\mathrm{MOH}^{29}$ Current estimations of different $\mathrm{TH}$ mobile application users in Saudi Arabia during the COVID-19 pandemic listed in "Goggle Play and Huawei AppGallery" are shown in Figure 2. There was remarkable higher user of Tawakkalna application reaching $>7$ million users up to date, this application developed to show the health status of its users through color codes at the highest degree of safety and privacy. It provide more than 30 services, also allows individuals to contribute to breaking the chain of infection by reporting infected cases or gatherings that violate the adopted precautionary measures. The use of TH mobile applications was a considerable facilitator that shows the Saudi health-care system had been fully prepared to deal with outbreaks such as COVID-19.

Ambulatory care in urology practice in Saudi Arabia has received attention since the emergence of the COVID19 pandemic and demands greater attention from stakeholders. It is crucial to reach out to urology outpatients, the majority of whom are elderly and have added health issues and complications. Such patients might be at a higher risk of COVID-19-associated complications. Patients with soft indications can be managed via telephone and they can be sent home with advice for care. Clinical patients with urological complaints that need to be seen on an urgent basis are advised to stay outside and can be brought straight into a clinic. Facemasks are given to such sufferers, preferably before entering. If possible, detached access for entry is offered for outpatients. Delivering urology outpatient services required regulating how triage was measured and applied to patients through means that do not depend on FTF care. Prescheduled outpatient appointments are screened and patients with nonurgent urological conditions are consulted via telephone and are prescribed treatment and asked to follow up. Patients in need of a physical examination are prioritized depending on the cause of the urological disease, such as suspected malignancy, obstructive uropathy, and immediate postoperative

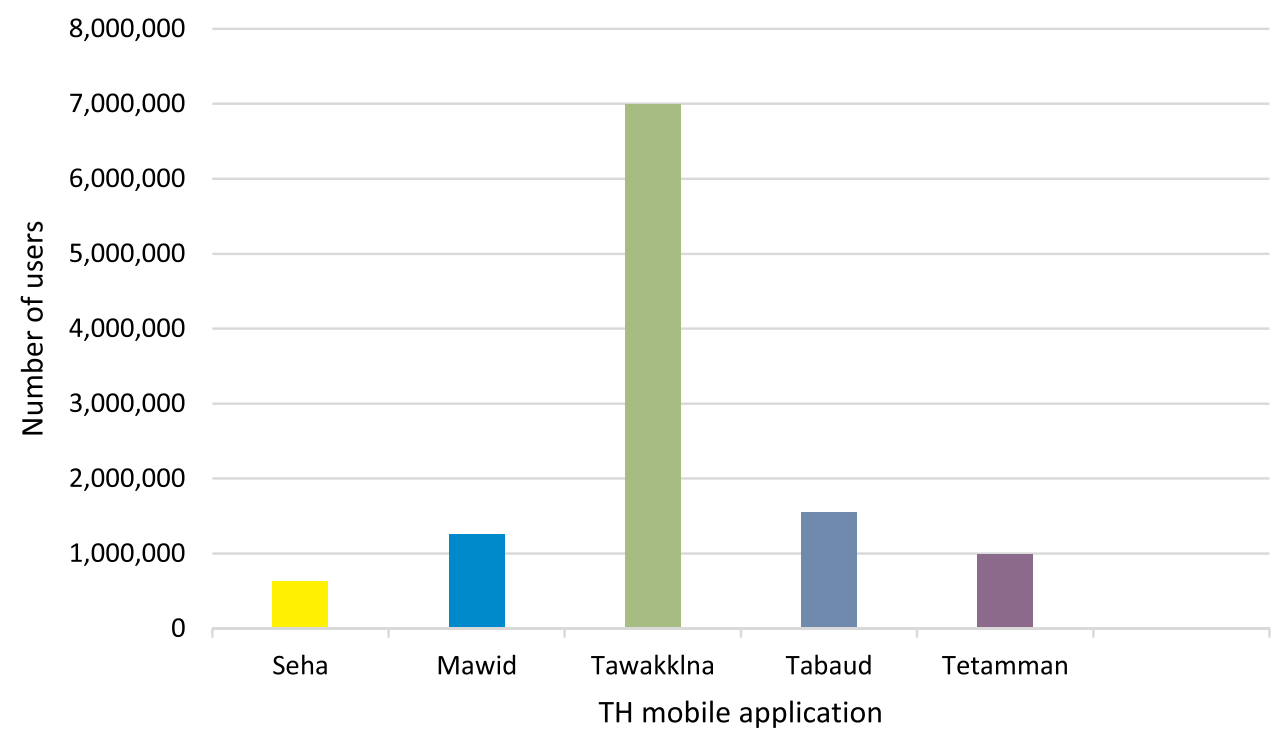

Figure 2 Numbers of current users of telehealth mobile applications during the COVID-19 pandemic in Saudi Arabia. Abbreviation: $\mathrm{TH}$, telehealth. 
cases. Patients presenting with macroscopic hematuria are placed on empirical therapy, thus delaying the recommended cystoscopy by one to two months. ${ }^{32}$ Prostatic biopsies and cystoscopy for patients at high risk of malignancy and extracorporeal shockwave lithotripsy for obstructive ureteric calculi are allowed to proceed. Indeed, various experts recommended managing cases with prostate imaging reporting and data system (PIRADS) score $4 / 5$ as planned, ${ }^{32}$ avoiding delays of more than six weeks for symptomatic patients as European Association of Urology (EAU) suggested. ${ }^{24}$

\section{Impact of COVID-I9 on Urological Procedures}

In retrospective cohort study, at the beginning of COVID-19 spread, data from China among 34 asymptomatic patients who underwent elective procedure reported that $44 \%$ required ICU and $20 \%$ died. $^{33}$ Mathematically, in very early stage of COVID-19 pandemic, the epidemiological model called Susceptible Infectious Exposed Recovered (SEIR) have been found as suitable model for COVID-19 spread predictions in Saudi Arabia. ${ }^{34}$ They predicted number of infections can be lowered to 0.4 million by September 2020. This study demonstrates that, with a stricter level of lockdowns, the COVID-19 curve can be effectively flattened in Saudi Arabi. After the detection of the first COVID-19 case in Saudi Arabia, strict nationwide policies were implemented to limit the transmission of the disease by implementing mass lockdown of cities, shutdown of schools and businesses, travel restrictions, implementation of testing protocols, and the use of novel technologies to trace suspected and confirmed cases. ${ }^{10,11}$ All these efforts helped to achieve a slow curve during the first two weeks of March. Elective surgeries were running as usual throughout the country. However, in late March, the number of cases increased dramatically despite these strict policies. Therefore, the Saudi $\mathrm{MOH}$ recommended rescheduling all elective non-urgent surgeries except for organ or life threatening and oncological cases. ${ }^{10,11}$ All governmental hospitals applied these recommendations, whereas surgical practice in the private sector continued at a much slower pace. These prompt and vigilant actions were taken by the $\mathrm{MOH}$ and all hospitals for many reasons, the main one was to prepare all the hospitals throughout the country to respond to any COVID-19 crisis scenario. ${ }^{10,11}$ In countries where the healthcare system collapsed and mortalities increased during the pandemic, the cause was mainly HCP exhaustion, the unavailability of ICU beds and ventilatory support facilities. $^{35,36}$ Therefore, these actions were taken to unburden as many of these facilities as possible, without exhausting the healthcare system with nonurgent stable cases that could be delayed. The Saudi $\mathrm{MOH}$ and SaudiCDC ${ }^{10,11}$ illustrated the plan and provided recommendations and guidelines to all the hospital and healthcare facilities in the country. Table 1 shows hospital admission criteria for COVID-19 patients while Table 2 shows ICU triage and admission criteria during the COVID 19 pandemic. Figure 3 shows algorithm infection control precautions and recommendations for elective surgeries during the COVID-19 pandemic. Emergency procedures that were life- or organ-threatening and those for opioid-dependent pain were continued all over the Kingdom. Regarding oncological procedures, most of the hospitals adapted the European Urology Association (EAU) guidelines that were developed by The Guidelines Office commissioned by the Rapid Reaction Group (GORRG) on 19 March 2020. ${ }^{24}$ In these guidelines,

Table I Summary of Hospital Admission Criteria Recommendation to Admit Any Patient Meets the Case Definition of Confirmed/ Suspected COVID-19 as Stated in MOH/SCDC Guidance (Version 1.3) Who is Symptomatic Plus Any of the Following Criteria

\begin{tabular}{|l|}
\hline $\begin{array}{l}\text { Hospital Admission Criteria for COVID-19 Patients (Version } \\
\text { I.I) }\end{array}$ \\
\hline Clinical or radiological evidence of pneumonia. \\
\hline Age $>65$ years. \\
\hline Low oxygen saturation SpO2 <94\% on room air. \\
\hline Acute respiratory distress syndrome (ARDS). \\
\hline Chronic pulmonary disease. \\
\hline Chronic kidney disease. \\
\hline History of comorbidities diabetes mellitus or/and hypertension. \\
\hline History of cardiovascular disease. \\
\hline Obesity (BMI $\geq 40$ ). \\
\hline $\begin{array}{l}\text { Use of biological (immunosuppressants) medications (eg, TNF } \\
\text { inhibitors, interleukin inhibitors, anti-B cell agents). }\end{array}$ \\
\hline History of organ transplant or another immunosuppression disease. \\
\hline History of active malignancy. \\
\hline Other co-illness that requires admission. \\
\hline
\end{tabular}


Table 2 Summary Highlight ICU Triage/Admission Criteria During the COVID 19 Pandemic (Version 2)

\section{ICU Triage Recommendations \\ ICU triage decisions are made according to patient conditions and the need for critical care monitoring and or intervention. \\ Patients to be admitted or discharged strictly on their potential to benefit from ICU care.}

Some over triage is more acceptable and preferable to under triage.

Transfer time of critically ill patients from the emergency department or ward bed to the ICU in less than or equal to I hour from the time of consultation if a bed is available.

If the ICU bed is unavailable, ICU physicians continue to deliver care for critical care patients in the emergency department or the ward with the help of the primary team.

The most senior ICU covering physician is responsible for making ICU triage decisions during routine or emergency conditions.

Scoring systems should not be used alone to determine the level of care or removal from higher levels of care.

Documentation of patients' wishes for the right of decision making and signing consents on their behalf when they are not able to do so should be done before admitting to ICU whenever possible.

Do Not Resuscitate (DNR) status should be determined before and during ICU stay to determine the need for critical care.

\section{Criteria for COVID I9 ICU Admission}

Need Invasive Mechanical Ventilation

Patient requiring more than 2 hours on Non-Invasive Ventilation (NIV) or High Flow Nasal Cannula (HFNC).

Respiratory Distress

- Need O2 > 6 LPM to maintain SpO2 > 92 or $\mathrm{PaO} 2>65$.

- Rapid escalation of oxygen requirement.

- Significant work of breathing, ie, Tachypnea.

Patient with hemodynamic instability despite initial conservative fluid resuscitation

Patient require vasopressor support.

Patient with a decreased level of consciousness.

Acidosis

- $\mathrm{ABG}$ with $\mathrm{pH}<7.3$ or $\mathrm{PCO} 2>50$ or above patient's baseline.

- Lactate $>2$

Patient with more than one organ failure.

Patient requires continuous renal preplacement therapy CRRT and cannot tolerate hemodialysis.

Patient with unstable vital signs not yet on vasopressors.

Patent with new ECG findings, including ischemia, arrhythmias, heart block (preferred to be in CCU if available). cases were classified and color-coded based on their level of priority from low priority to emergency (Figure 4). Accordingly, all cases were assigned to their corresponding level of priority and a decision was made to postpone or to proceed. When any elective surgery was scheduled, the policy mandated screening all patients with a nasal and/or oropharyngeal swab within 48 hours prior to surgery while still an outpatient. For patients with respiratory symptoms and in need of an emergency surgery, a COVID-19 test was performed, and the patients were treated as a positive case to minimize the risk of spreading the infection. When operating on a COVID-19 positive case, all necessary precautions were advised. ${ }^{24}$ The number of working staff in the operating room was kept to the minimum.

With regard to renal transplant cases, tertiary care centers performing such surgeries followed the Position Statement on Organ Transplant and Donation during Coronavirus Disease (COVID-19) Pandemic issued by the Saudi Center for Organ Transplantation (SCOT). ${ }^{10}$ All potential donors (living and cadaveric) were tested with a nasopharyngeal and oropharyngeal swab for COVID-19. If the donor was from a high-risk region within the country, then another confirmatory swab was taken. Recipients were only cleared after having a negative swab (two negative swabs if they were from a high-risk area). Very detailed protocols were also constructed to manage early post-transplant recipients who were suspected or confirmed to have COVID-19.

The CovidSurg Collaborative group recently published an article estimating the number of cancelled or postponed operations during the COVID-19 pandemic. ${ }^{37}$ In urology, 458,151 (36.6\%) cancer surgeries and 2,492,604 (81.7\%) benign surgeries were estimated to have been cancelled over the 12-week period of peak disruption. They concluded that if the surgical capacity increased by $20 \%$ after the pandemic, it would take about 45 weeks to clear the backlog of operations resulting from the COVID-19 disruption.

Real-life data from three Italian academic urological centers $^{38}$ was evaluated as to how many different urooncologic procedures can be deferred in emergency times. To guide prioritization strategies during the COVID-19 pandemic, they assessed and provided a reallife overview of the yearly proportion of high-priority major uro-oncologic procedures, 2387 patients were included between 2018 to 2019, approximately two-thirds $(67.8 \%)$ of patients do not require high-priority surgery and can be postponed. Only $32.3 \%$ were classified as high 
Elective surgery during Covid-19 Pandemic

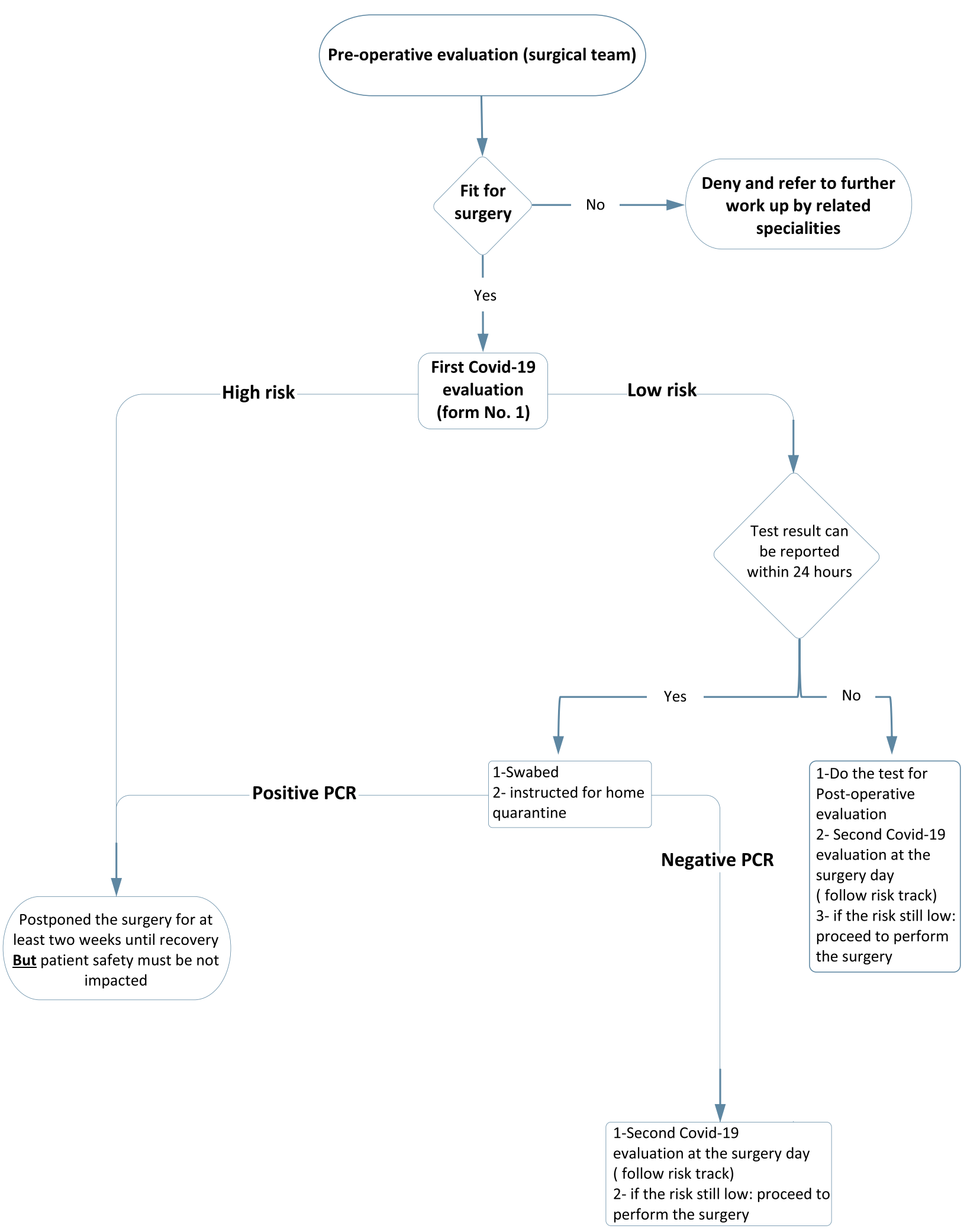

Figure 3 Infection control precautions and recommendations for elective surgeries during the COVID-19 pandemic in Saudi Arabia.

Note: Copyright @2020. Reproduced from COVID-19 Coronavirus Disease Guidelines. Saudi center for disease prevention and control (SaudiCDC - Weqaya). Available from: https://covid 19.cdc.gov.sa/. Accessed October 20, 2020." 


\begin{tabular}{|c|c|c|c|c|}
\hline $\begin{array}{l}\text { Priority } \\
\text { category }\end{array}$ & Low priority & $\begin{array}{l}\text { Intermediate } \\
\text { priority }\end{array}$ & High priority & Emergency \\
\hline Definition & $\begin{array}{l}\text { Clinical harm very } \\
\text { unlikely if } \\
\text { postponed for } 6 \\
\text { mo }\end{array}$ & $\begin{array}{l}\text { Clinical harm } \\
\text { possible if } \\
\text { postponed for 3-4 } \\
\text { mo, but unlikely }\end{array}$ & $\begin{array}{l}\text { Clinical harm likely if } \\
\text { postponed for }>6 \mathrm{wk}\end{array}$ & $\begin{array}{l}\text { Life-threatening } \\
\text { situation; likely to } \\
\text { have presented via } \\
\text { A\&E despite the } \\
\text { current pandemic }\end{array}$ \\
\hline
\end{tabular}

Figure 4 Color-coded risk stratification tool show levels of priority of urological cases during COVID-I9 pandemic period proposed by GORRG.

Note: Copyright (2020. Elsevier B.V. Reproduced from Ribal MJ, Cornford P, Briganti A, et al. European Association of Urology Guidelines Office Rapid Reaction Group: An Organisation-wide Collaborative Effort to Adapt the European Association of Urology Guidelines Recommendations to the Coronavirus Disease 2019 Era. Eur Urol. 2020;78(I):2I-28. doi:10.1016/j.eururo.2020.04.056. ${ }^{24}$

Abbreviation: A\&E, accident and emergency department.

priority. Approximately $26.4 \%$ were considered at high perioperative risk with American Society of Anesthesiologists score (ASA) $\geq 3$ and may create challenges in resource allocation in critical periods such as the current COVID-19 pandemic. For high priority patients, the use of alternative treatment modalities may be even more important to further reduce the potential need for ICU given the limited resources, as the authors suggested. These data help other urology communities worldwide in adapting their management strategies for the inflow of urooncologic patients considering the expected shortage in resources caused by the COVID-19 pandemic. The lesson from such analysis is that careful selection of surgical candidates is key during the COVID-19 pandemic.

These estimations note the huge forthcoming burden on the urological practice in Saudi Arabia and around the world. Nationwide efforts are presently being implemented to facilitate clearing all the delayed and cancelled cases. Recently, systemic review showed multiple national and international guidelines and recommendations for urologists to prioritize the urological care during the COVID19 pandemic. $^{39}$ Among those guidelines the differences were minimal, they able to look over the evidence level and recommendations grade, they proposed triage color codes for emergency and elective procedures illustrated in
Figure 5. It is important to utilize the level of evidence to guide and provide better health care in such pandemic time. All recommendations represented in this review ${ }^{39}$ were expert opinions, with three specifically endorsed by professional societies, only the UAE guidelines provided evidence-based levels of evidence (mostly level 3 evidence). They concluded recommendations as following; with lower-risk tumors to apply the active surveillance approach (eg, low-risk PCa, low-grade bladder cancer, and small renal masses), in some advanced cases the systemic therapies (neoadjuvant or adjuvant treatments) or cytoreductive nephrectomy can be omitted. For uncomplicated ureteral stones, they propose use of medical expulsive therapy. In cases with high risk of urosepsis or functional sequelae like severe infection and/or obstruction of the kidneys must be treated accordingly. Even at a time of high workload burden on the health system, urological emergencies like active hematuria, infected implants, or postoperative bleeding and ischemic complications must be treated immediately.

\section{Challenges for Urology Residency Training}

Worldwide, at time of writing, Italy was one of the countries reporting the highest number of deaths due to COVID-19. Italy faced a serious challenge during the

\begin{tabular}{|c|l|}
\hline 0 & Emergency. Survivorship compromised if surgery not performed within hours. \\
\hline 1 & $\begin{array}{l}\text { Proceed as planned, do not postpone. } \\
\text { Survivorship compromised if surgery not performed within days. } \\
\text { Usually resources exhausted. }\end{array}$ \\
\hline 2 & $\begin{array}{l}\text { Consider delay } 1 \text { month. } \\
\text { Patient condition can deteriorate or survivorship compromised if surgery not performed } \\
\text { within } 30 \text { days. } \\
\text { Or proceed as planned if COVID trajectory not in rapid escalation phase. }\end{array}$ \\
\hline 3 & $\begin{array}{l}\text { Safe to delay 1-3 months. } \\
\text { Or proceed as planned if COVID trajectory not in rapid escalation phase. }\end{array}$ \\
\hline 4 & Safe to delay > 3 months. \\
\hline
\end{tabular}

Figure 5 Proposed triage color codes for emergency and elective procedures during the COVID-19 pandemic.

Note: Copyright @2020. Elsevier B.V. Reproduced from Heldwein FL, Loeb S, Wroclawski ML, et al. A Systematic Review on Guidelines and Recommendations for Urology Standard of Care During the COVID-19 Pandemic. Eur Urol Focus. 2020;6(5):1070-1085. doi:10.1016/j.euf.2020.05.020. ${ }^{39}$ 
COVID-19 pandemic, and their healthcare system was near collapse due to the aggressive spread of the infection. Unfortunately, as a result, many HCPs in Italy were infected or died. ${ }^{40,41}$ Rapidly, this current situation across all hospitals in Saudi Arabia led to a significant decrease in clinical and surgical practice. As a result, physicians in training (PIT) might be critically affected. ${ }^{42}$

During the pandemic, in a cross-sectional study conducted in Saudi Arabia among 240 trainees (residents and fellows $)^{42}$ showed $43 \%$ had direct contact with COVID-19 patients, regarding the proper use of PPE $43.8 \%$ had appropriate training. Only $2.9 \%$ had been infected by COVID-19. They reported significant reduction in training activities (84.6\%) and surgical exposure (97\%) due to the current COVID-19 pandemic.

A study from Italy $^{41}$ reported the first nationwide survey on urology residency training during the pandemic period, comparing the routine involvement of residents before and after the pandemic period regarding their training in clinical and surgical duties. They found that during the pandemic, urology residents experienced a severe reduction $>40 \%$ and complete suppression $>80 \%$ in clinical and surgical activities compared to before the pandemic.

As with all other surgical specialties, in Saudi Arabia, urology practice was negatively affected by the COVID-19 pandemic. Cancelling and deferring most of the surgical cases was not in the interest of urology residents. Centers and academic institutes with a high resident load suffered the most, with few emergency and cancer surgeries being performed during the pandemic. Urology has a wide variety of subspecialties, all of which were placed on hold except for uro-oncology cases or urological emergencies. ${ }^{39}$ Policies that mandated minimizing the number of healthcare personnel in the operating theater also led to a lower chance of surgical hands-on training. With regards outpatient practice, urology residents continued to use TM on a virtual basis throughout the country. This led to a decreased amount of direct exposure to patients, and urological surgical procedures, resulted in having plenty of free time during the outbreak which increased chances of available time for teaching though e-learning platforms. Until that moment, no urology residents had been asked to crossover with other medical or ICU services. However, they were on standby to provide help whenever needed.

As is evident, learning and studying during the COVID-19 pandemic for residents was challenging. Data from Italy ${ }^{44}$ showed majority of Italian urology residents experienced slowdown in their learning process and they spend at least two hours per day $(85.2 \%)$, while few have four or more hours (32.5\%). Study habits of urology residents in Saudi Arabia have been studied, ${ }^{45}$ the study conducted a year before the pandemic time showed $37.3 \%$ spent 2-5 hours weekly. Juniors read significantly more than seniors $(P=0.034)$. The most useful study source comprised digests and study notes, followed by the Campbell-Walsh Urology textbook and American Urological Association (AUA)/EAU guidelines books. The AUA/EAU guidelines were useful information source for seniors $(P=0.001)$. These results may have changed during the pandemic time among urology residents, however based on Italian study, ${ }^{44}$ the studying methods may be varied and might be improved with using smart-learning, which can trigger the trainees interest to improve their knowledge and reading habits, especially during such pandemic time when experiencing a severe reduction in daily working hours and duties. For training programs, implementation of smart technology should be adopted during such pandemic, to secure continuity in the learning curve processes. $^{42,43}$

In the current pandemic situation, necessity should be transformed into a valued future opportunity. The smartlearning can be implemented routinely as a useful tool for residency training programs and daily urology practice. ${ }^{43}$ For the sake of residents' learning curve improvement during the COVID-19, authors suggested various smartlearning technologies and their possibility of application includes pre-recorded videos on-demand, webinar, journals club via social media, podcast, clinical staff rounds and simulation. ${ }^{43}$ During the pandemic time, urology residents from Italy ${ }^{44}$ reported the most useful resources for smart learning as following: updated guidelines, surgical videos, clinical case discussion, dedicated lectures, and journal clubs $89.5 \%, 84.3 \%, 77.2 \%, 67 \%$ and $57.8 \%$, respectively.

One positive side of the COVID-19 pandemic was the enormous growth in online teaching activities and seminars. Weekly teaching activities increased to two or three times a week, with more intense scientific content and a higher number of consultants attending and moderating these activities. Local association and groups (eg, Saudi Urological association [SUA] and Saudi Society of Men's Health [SSMH]) made tremendous efforts to arrange and conduct national and international online webinars and lectures in all urological subspecialties with renowned speakers from all over the world. The Annual Saudi 
Urology Board Review Course, conducted and delivered yearly by the SUA, was converted into an online course to prepare the residents in their last year for their final board exam. This provided a unique educational environment that boosted the academic performance of residents.

Surprisingly, with regard to studying and reading during the pandemic, residents and fellows from Saudi Arabia had adequate time (41.3\%), but psychologically feel unprepared $(43.3 \%){ }^{42}$ Studies conducted on psychological and mental health care training during COVID-19 pandemic found HCPs, especially juniors, are not well prepared to deal and cope with such global health crisis. ${ }^{46,47}$ Aldossary ${ }^{42}$ reported significant impact of the COVID-19 pandemic in board examination among training program registered at Saudi Commission for Health Specialties (SCFHS), they reported that the PIT were significantly unprepared psychologically for studying $(\mathrm{P}<0.001)$ and they feel stressed about upcoming exams $(\mathrm{P}<0.001)$. When examination delays happen due to pandemic situation, the authors recommended to continue studying and revision using webinars, podcasts, prerecorded sessions, and social media. If possible, routine activities such as ground rounds, dedicated teaching lectures, and journal clubs may continue through webinars.

The SCFHS, the supervising training body in Saudi Arabia, asked the scientific committees of each training program to formulate recommendations for how the SCFHS should deal with the training program during the pandemic. The urology scientific committee provided specific recommendations for each aspect of training. Rotations were rearranged or postponed, exam dates were reset, and evaluation methods were reestablished. All these efforts were undertaken to minimize the effect of the COVID-19 pandemic on urology training. These recommendations were to be revisited after three months according to the overall situation of the pandemic.

The SCFHS launched two unique initiatives. Firstly, Imtinan (gratitude) ${ }^{48}$ was a gesture of appreciation for the effort from all healthcare workers and especially residents in the fight against COVID-19. It enabled registered healthcare practitioners to extend their registration for a period of four months free of charge without the need to provide extra continuous medical education (CME) hours that are normally needed for the registration for more than 80,000 health practitioners up to date. Over 30,000 trainees received a prerecorded phone call from the Secretary General of the SCFHS thanking them for their efforts during these difficult times. More than 20,000 appreciation letters from the SCFHS were sent to the families of healthcare workers. More than
19,000 gifts to families and friends who supported the practitioners were given as a gesture of appreciation for their effort on their behalf.

Another initiative by the SCFHS was Da'em (support), ${ }^{49}$ a psychological supportive service helping health practitioners who were suffering from stress, burnout, and depression. Around 53 professionals (psychiatrists and psychologists) provided mental and psychological support; health practitioners included trainees, with therapeutic sessions, lectures, and webinars. All these efforts and initiatives were intended to protect the physical and mental wellbeing of health practitioners all over the country. Currently, Da'em services are available for more than 500,000 health practitioners.

\section{Urology Education and Activities During the COVID-I9 Pandemic}

Educational institutions worldwide experienced disruptions in their operations and were forced to adopt new methods of addressing the challenges of the situation. The COVID-19 outbreak interrupted all the traditional forms of FTF communication, eg, monthly and yearly scientific meetings, dedicated hospital-based lectures, conferences, and journal clubs. As a result of the pandemic, surgeons, especially those in the urology specialty, had a limited presence in operating rooms due to the high cancellation rate of elective procedures, and they found an opportunity for online learning during the lockdown and quarantine period.

In Saudi Arabia, many urological societies provide invaluable support to the urologic community, for example, the SUA and the SSMH. These societies are considered two of the premiere urology associations in the Middle East. These societies took the lead in terms of embracing and developing sustainable alternatives to traditional educational activities during the COVID-19 pandemic and helped in transitioning to distance learning to ensure urology education continued without interruption. From the spread of the pandemic, the urological society created an events calendar on the official website for upcoming live webinars. These activities were announced through the official website and through official social media platforms. These events were conducted on online streaming media platforms and were simultaneously recorded and broadcast in real time. The webinars were scheduled for times convenient to the presenters and audience, and the recorded sessions were made available on the official website for those not able to catch the live sessions. They used different e-learning platforms and applications such as Zoom ${ }^{\circledR}$ (Zoom, San Jose, CA, 
USA), Cisco Webex ${ }^{\circledR}$ (Cisco Systems, Milpitas, California, USA), and GoToWebinar ${ }^{\circledR}$ (LogMeIn, Boston, Massachusetts, USA). The most common platform used was Zoom ${ }^{\circledR}$, which provides structural advanced encryption software (AES) with 256-bit encryption. These platforms were protected by a substantially higher level of security compared to the original web-based platforms. ${ }^{50}$

These societies created a huge number of webinars in the form of online interactive scientific lectures, focusing on a variety of advanced urological topics in different urological subspecialties from early in the pandemic, with contributions from local and international pioneer urologists and men's health experts. In addition, cooperation occurred with local and international urological organizations to provide educational activities of clinical excellence, delivered in a safe and effective manner in the form of e-learning.

During the pandemic, efforts were made to continually improve the urology community. Recently, the urology department at Majmaah University officially launched the Saudi Pelvic Floor Surgery Society. This reflects how the urology community evolved even with the challenges associated with the pandemic.

With regards to patient education of common urological conditions during pandemic time, the SUA, in cooperation with official foundation of AUA "Urology Care foundation", launched initial phase of Arabic language translation of some educational materials provided to general public in Saudi Arabia and distributed through the official website of SUA and their social media accounts, supporting patient information and education during pandemic crisis.

Some urologists in our country were invited onto TV shows as advocates for society health education regarding COVID-19, providing advice regarding urology healthcare during the pandemic period. Others started communicating with the Saudi population through educational broadcasts on social media to reinforce the need to stay at home and comply with the quarantine restrictions, as well as to educate people on how to deal with urological emergencies and special urological conditions.

Finally, the quick move to online education posed many challenges. However, there were lessons to be learned, providing a chance for the urology community to adapt and improve our forms of communication for the future. Adoption of technology is important not just in clinical practice, but also in communication and administration during these pandemic times. Overcoming the current global health crisis will be difficult, but through creative approaches in teaching, we think that as urologists we will move forward to improve and adapt. Our community will successfully move through the COVID-19 period with a sustained focus on our community wellbeing and academic success.

\section{Impact of the COVID-I 9 Pandemic and Its Related Consequences on the Future of Urological Services}

Rapidly, the COVID-19 pandemic necessitated immediate changes in our practice and has had lasting effects on our health care system. Most of these changes will add value to our current and future care, creating opportunities to improve our knowledge and response to such pandemic era.

A single center study ${ }^{51}$ evaluated triage and postponed elective cases totaling 204 urologic cases during the Covid19 pandemic; over the ensuing 12 weeks they used different surgical prioritization scoring systems to establish surgical prioritization strategies during the pandemic, their observation suggest potential for different advantages and drawbacks of these parameters depending on the strategy chosen. TimeSensitive Procedures (MeNTS) Scoring System called Questionnaire-based Surgical Prioritization (QSP), expert opinion-base (EOP), and individual surgeon-based (ISP) approaches result in significantly different case prioritization. QSP did not meaningfully stratify urologic cases, and EOP and ISP frequently disagreed. Such parameters should accordingly be tailored to existing conditions at individual centers, cities and geographical regions. They suggested such findings should be integrated at the time of future disaster planning.

A review addressed dramatic changes in the care provided to many patients with genitourinary cancers during the COVID-19 pandemic. $^{52}$ They found these changes offer opportunities to improve access, delivery, and the value of care to such patients and for their families. These opportunities can be utilized in future as a great experience learned from current pandemic, like transition to TM, changes in threshold or delays in many treatments, and an opportunity to reimagine patient care to maintain safety and improve value moving forward (Figure 6).

To get maximum benefits from such emergency times, urologists must strive to reshape their role in the future. In attempt forecasting the future of Urology practice during the pandemic period, groups of urologist researchers from Europe $^{23}$ extensively reviewed the recommendations released by a total of 13 international and European national urological associations/societies (UASs) with regard to urological procedures prioritization strategies, use of minimally 


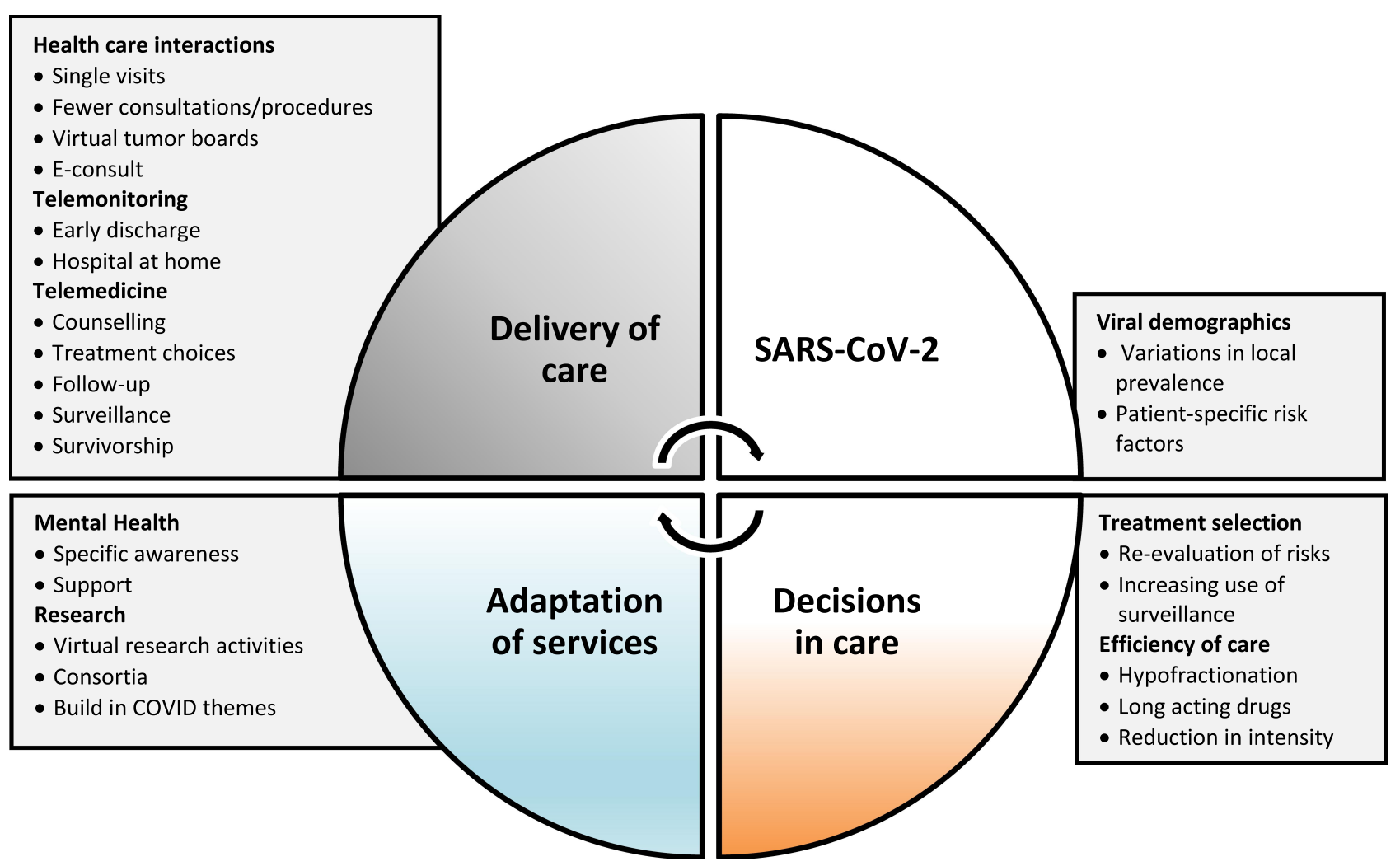

Figure 6 Proposed strategies post-COVID-19 pandemic care for genitourinary cancer patients.

Note: Copyright (C2020. Elsevier B.V. Reproduced from Wallis CJD, Catto JWF, Finelli A, et al. The Impact of the COVID-19 Pandemic on Genitourinary Cancer Care: Reenvisioning the Future. Eur Urol. 2020;78(5):731-742. doi:10.1016/j.eururo.2020.08.030.52

invasive surgery (MIS) and TM. These recommendations may have possible implications for urology practice in both the forthcoming "adaptive" and the future "chronic" period of the COVID-19 pandemic. In the outpatient setting, prostate biopsies and elective surgeries (eg, benign urological conditions) are most likely to impact the future urology burden workload. While in the inpatient setting, elective procedures for lower-risk $\mathrm{PCa}$ and kidney cancers, nonobstructing urolithiasis, and $\mathrm{BPH}$ are the most pertinent contributors to this burden. There were concerns regarding the COVID-19 transmission risk through surgical smoke production during MIS. Some UASs recommended special precautions to perform such procedures, urological MIS should be performed according to the most confident approach by surgeon, use of specific surgical smoke evacuator and $\mathrm{CO} 2$ insufflation with a closed system and proper aerosolized particles filtration, apply possible allowed lowest intra-abdominal pressure, and to reduce surgical smoke production they advised to decrease electrocautery power setting. ${ }^{23}$ The use of TM showed potential role to optimize resources and procedures prioritization in the current and future pandemic. ${ }^{23}$

\section{Conclusions}

A tremendous amount of work was undertaken in the face of the COVID-19 pandemic in Saudi Arabia. Worldwide, the urologists played a part in confronting the crisis and had a role to play in serving society and public health.

Many lessons can be learned from this pandemic. Early implementation of Telemedicine and carfeul surgical procedures prioritization provided favorable outcomes. Among urologist, there was valuable time and good opportunity for transformation of traditional teaching into e-learning platforms. Professional communication with immediate intervention along with establishment of alternative practical methods to maintain the patient care and learning process are crucial. The exchange and progress of scientific information is essential to ensure support for healthcare providers and patient safety in such pandemic era. 


\section{Abbreviations}

AES, Advanced Encryption Software; ARIMA, Autoregressive Integrated Moving Average; AUA, American Urological Association; BPH, Benign prostatic hyperplasia; COVID-19, Coronavirus Disease of 2019; CME, Continuous Medical Education; EAU, European Association of Urology; ESSM, European Society for Sexual Medicine; FFP, Filtering facepiece; FTF, Face-toface; GORRG, Guidelines Office commissioned a Rapid Reaction Group; HCP, Healthcare Providers; ICU, Intensive Care Unit; $\mathrm{MOH}$, Ministry of Health; PPE, Personal Protective Equipment; PIT, physicians in training; PCa, Prostate cancer; PIRADS, Prostate Imaging Reporting and Data System; SMS, short message service; SUA, Saudi Urological Association; SSMH, Saudi Society of Men's Health; SCOT, Saudi Center for Organ Transplantation; TH, Telehealth; TM, Telemedicine; WHO, World Health Organization.

\section{Acknowledgments}

The authors would like to thank the Deanship of Scientific Research at Majmaah University for supporting this work under Project Number R-2021-13.

\section{Disclosure}

The authors report no conflicts of interest in this work.

\section{References}

1. Cascella M, Rajnik M, Cuomo A, Dulebohn SC, Di Napoli R. Features, Evaluation, and Treatment of Coronavirus (COVID-19). In: Stat Pearls. Treasure Island (FL): StatPearls Publishing; 2020 August 10.

2. Lai CC, Shih TP, Ko WC, Tang HJ, Hsueh PR. Severe acute respiratory syndrome coronavirus 2 (SARS-CoV-2) and coronavirus disease-2019 (COVID-19): the epidemic and the challenges. Int J Antimicrob Agents. 2020;55(3):105924. doi:10.1016/j.ijantimicag.2020.105924

3. Perlman S, Netland J. Coronaviruses post-SARS: update on replication and pathogenesis. Nat Rev Microbiol. 2009;7(6):439-450. doi:10.1038/nrmicro2 147

4. Cui J, Li F, Shi ZL. Origin and evolution of pathogenic coronaviruses. Nat Rev Microbiol. 2019;17(3):181-192. doi:10.1038/s41579-0180118-9

5. World Health Organization. Coronavirus disease (COVID-2019) situation reports. Available from: https://www.who.int/emergencies/diseases/ novel-coronavirus-2019/situation-reports. Accessed October 20, 2020.

6. Alzahrani SI, Aljamaan IA, Al-Fakih EA. Forecasting the spread of the COVID-19 pandemic in Saudi Arabia using ARIMA prediction model under current public health interventions. J Infect Public Health. 2020;13(7):914-919. doi:10.1016/j.jiph.2020.06.001

7. Atique S, Itumalla R. Hajj in the Time of COVID-19. Infect Dis Health. 2020;25(3):219-221. doi:10.1016/j.idh.2020.04.001

8. Ministry of Health. Covid19 Command and Control Center CCC, The National Health Emergency Operation Center NHEOC. Available from: https://covid19.moh.gov.sa/. Accessed October 20, 2020.
9. Saudi Worldometer. Arabia. Coronavirus cases; 2020. Available from: https://www.worldometers.info/coronavirus/country/saudiarabia/. Accessed October 20, 2020.

10. COVID-19 Guidelines. MOH Publications. MOH Portal. Available from: https://www.moh.gov.sa/en/Ministry/MediaCenter/ Publications/Pages/covid19.aspx. Accessed October 20, 2020.

11. COVID-19 Coronavirus Disease Guidelines. Saudi center for disease prevention and control (SaudiCDC - Weqaya). Available from: https://covid19.cdc.gov.sa/. Accessed October 20, 2020.

12. MOH. Call (937) Service Center for Inquires about Novel Coronavirus. Available from: https://www.moh.gov.sa/en/Ministry/ MediaCenter/News/Pages/News-2020-02-29-002.aspx. Accessed October 20, 2020.

13. MOH Portal. Health Awareness, (Tetamman) Clinics at Healthcare Centers and Hospitals. Available from: https://www.moh.gov.sa/en/ HealthAwareness/Tataman-Clinics/Pages/default.aspx. Accessed October 20, 2020.

14. MOH Portal. Awareness Plateform, Various Topics, COIVD-19. Available from: https://covid19awareness.sa/en/home-page. Accessed October 20, 2020.

15. Al Sulais E, Mosli M, AlAmeel T. The psychological impact of COVID-19 pandemic on physicians in Saudi Arabia: a cross-sectional study. Saudi J Gastroenterol. 2020;26(5):249-255. doi:10.4103/sjg.SJG_174_20

16. Teoh JY, Ong WLK, Gonzalez-Padilla D, et al. A Global Survey on the Impact of COVID-19 on Urological Services. Eur Urol. 2020;78 (2):265-275. doi:10.1016/j.eururo.2020.05.025

17. COVID-19: Infection prevention and control / WASH. Infection prevention and control during health care when novel coronavirus (nCoV) infection is suspected. Available from: https://www.who.int/ publications/i/item/10665-331495. Accessed October 20, 2020.

18. WHO. Clinical management of severe acute respiratory infection when novel coronavirus $(2019-\mathrm{nCoV})$ infection is suspected. interim guidance. WHO 2020. https://www.who.int/publications/i/item/clini cal-management-of-covid-19. Accessed October 20, 2020.

19. Wang D, Hu B, Hu C, et al. Clinical Characteristics of 138 hospitalized patients with 2019 novel coronavirus-infected pneumonia in Wuhan, China. JAMA. 2020;323(11):1061-1069. doi:10.1001/ jama.2020.1585

20. Bashshur R, Doarn CR, Frenk JM, Kvedar JC, Woolliscroft JO. Telemedicine and the COVID-19 Pandemic, Lessons for the Future. Telemed $J$ E Health. 2020;26(5):571-573. doi:10.1089/ tmj.2020.29040.rb

21. American Medical Association. COVID-19 Recent and Ongoing Advocacy Efforts. Available from: https://www.ama-assn.org/deliver ing-care/public-health/covid-19-amas-recent-and-ongoing-advocacyefforts. Accessed October 20, 2020.

22. Marcin JP, Rimsza ME, Moskowitz WB, Committee On Pediatric Workforce. The Use of Telemedicine to Address Access and Physician Workforce Shortages. Pediatrics. 2015;136(1):202-209. doi: 10.1542/peds.2015-1253

23. Amparore D, Campi R, Checcucci E, et al. Forecasting the Future of urology practice: a comprehensive review of the recommendations by international and european associations on priority procedures during the COVID-19 pandemic. Eur Urol Focus. 2020;6(5):1032-1048. doi:10.1016/j.euf.2020.05.007

24. Ribal MJ, Cornford P, Briganti A, et al. European association of urology guidelines office rapid reaction group: an organisation-wide collaborative effort to adapt the European association of urology guidelines recommendations to the coronavirus disease 2019 Era. Eur Urol. 2020;78(1):21-28. doi:10.1016/j.eururo.2020.04.056

25. Rodriguez Socarrás M, Loeb S, Teoh JY, et al. Telemedicine and Smart Working: recommendations of the European Association of Urology [published online ahead of print, 2020 Jul 9]. Eur Urol. 2020;S0302(20):30460-30467. doi:10.1016/j.eururo.2020.06.031 
26. O’Connor M, Asdornwised U, Dempsey ML, et al. Using Telehealth to Reduce All-Cause 30-Day Hospital Readmissions among Heart Failure Patients Receiving Skilled Home Health Services. Appl Clin Inform. 2016;7(2):238-247. doi:10.4338/ACI-2015-11-SOA-0157

27. Novara G, Checcucci E, Crestani A, et al. Telehealth in urology: a systematic review of the literature. how much can telemedicine be useful during and after the COVID-19 Pandemic? [published online ahead of print, 2020 Jun 18]. Eur Urol. 2020;S0302(20):30454. doi:10.1016/j.eururo.2020.06.025

28. Digital Health Strategy Update 2018. Available from: https://www. moh.gov.sa/Ministry/vro/eHealth/Documents/MoH-Digital-HealthStrategy-Update.pdf. 20, 2020.

29. Alghamdi SM, Alqahtani JS, Aldhahir AM. Current status of telehealth in Saudi Arabia during COVID-19. J Family Community Med. 2020;27(3):208. doi:10.4103/jfcm.JFCM_295_20

30. Alruzaiza SA, Mahrous RM. Assessment of knowledge, attitude, and practice on level of awareness among pediatric emergency department visitors-Makkah City, Saudi Arabia: cross-sectional study. Int J Psychosoc Rehabil. 2020;24:5186-5202.

31. Alshammari F. Perceptions, preferences and experiences of telemedicine among users of information and communication technology in Saudi Arabia. J Health Inform Dev Ctries. 2019;13:1.

32. USANZ. Guidelines for urological prioritisation during COVID-19. Available from: https://www.usanz.org.au/news-updates/ourannouncements/usanz-announces-guidelines-urological-prioritisationcovid-19. Accessed October 20, 2020.

33. Lei S, Jiang F, Su W, et al. Clinical characteristics and outcomes of patients undergoing surgeries during the incubation period of COVID-19 infection. E Clin Med. 2020;21:100331. doi:10.1016/j. eclinm.2020.100331

34. Alrashed S, Min-Allah N, Saxena A, Ali I, Mehmood R. Impact of lockdowns on the spread of COVID-19 in Saudi Arabia. Inform Med Unlocked. 2020;20:100420. doi:10.1016/j.imu.2020.100420

35. Liu Q, Luo D, Haase JE, et al. The experiences of health-care providers during the COVID-19 crisis in China: a qualitative study. Lancet Glob Health. 2020;8(6):e790-e798. doi:10.1016/S2214-109X(20)30204-7

36. Armocida B, Formenti B, Ussai S, Palestra F, Missoni E. The Italian health system and the COVID-19 challenge. Lancet Public Health. 2020;5(5):e253. doi:10.1016/S2468-2667(20)30074-8

37. COVIDSurg Collaborative. Elective surgery cancellations due to the COVID-19 pandemic: global predictive modelling to inform surgical recovery plans [published online ahead of print, 2020 May 12]. $\mathrm{Br}$ J Surg. 2020. doi:10.1002/bjs.11746

38. Campi R, Amparore D, Capitanio U, et al. Assessing the burden of nondeferrable major uro-oncologic surgery to guide prioritisation strategies during the COVID-19 pandemic: insights from three Italian high-volume referral centres. Eur Urol. 2020;78(1):11-15. doi:10.1016/j.eururo.2020.03.054

39. Heldwein FL, Loeb S, Wroclawski ML, et al. A systematic review on guidelines and recommendations for urology standard of care during the COVID-19 pandemic. Eur Urol Focus. 2020;6(5):1070-1085. doi:10.1016/j.euf.2020.05.020
40. Armocida B, Formenti B, Ussai S, Palestra F, Missoni E. The Italian health system and the COVID-19 challenge. Lancet Public Health. 2020;5(5):e253. doi:10.1016/S2468-2667(20)30074-8

41. Amparore D, Claps F, Cacciamani GE, et al. Impact of the COVID-19 pandemic on urology residency training in Italy. Minerva Urol Nefrol. 2020;72(4):505-509. doi:10.23736/S03932249.20.03868-0

42. Balhareth A, AlDuhileb MA, Aldulaijan FA, Aldossary MY. Impact of COVID-19 pandemic on residency and fellowship training programs in Saudi Arabia: a nationwide cross-sectional study. Ann Med Surg. 2020;57:127-132. doi:10.1016/j.amsu.2020.07.025

43. Porpiglia F, Checcucci E, Amparore D, et al. Slowdown of urology residents' learning curve during the COVID-19 emergency. BJU Int. 2020;125(6):E15-E17. doi:10.1111/bju.15076

44. Claps F, Amparore D, Esperto F, et al. Smart learning for urology residents during the COVID-19 pandemic and beyond: insights from a nationwide survey in Italy [published online ahead of print, 2020 May 20]. Minerva Urol Nefrol. 2020;10:23736.

45. Alkhamees M, Al-Zahrani MA, Almutairi S, Alkanhal H, Almuhaideb M, Abumelha SM. Study habits of urology residents in saudi arabia: identifying defects and areas for curricular development - a trainee-based survey. Adv Med Educ Pract. 2020;11:707-715. doi:10.2147/AMEP.S269059

46. Xiang YT, Yang Y, Li W, et al. Timely mental health care for the 2019 novel coronavirus outbreak is urgently needed. Lancet Psychiatry. 2020;7(3):228-229. doi:10.1016/S2215-0366(20)30046-8

47. Lima CKT, Carvalho PMM, Lima IAAS, et al. The emotional impact of Coronavirus 2019-nCoV (new Coronavirus disease). Psychiatry Res. 2020;287:112915. doi:10.1016/j.psychres.2020.112915

48. Saudi Commission for Health Specialties. Imtinan initiative. Available from: https://www.scfhs.org.sa/en/Gratitude/Pages/default. aspx. Accessed October 20, 2020.

49. Saudi Commission for Health Specialties. Da'em service. Available from: https://www.scfhs.org.sa/en/eservices/Practitioners/Pages/ Daem-practitioners.aspx. Accessed October 20, 2020.

50. Connor MJ, Winkler M, Miah S. COVID-19 pandemic - is virtual urology clinic the answer to keeping the cancer pathway moving? BJU Int. 2020;125(6):E3-E4. doi:10.1111/bju.15061

51. Cohn JA, Ghiraldi EM, Uzzo RG, Simhan J. A critical appraisal of the acs "medically-necessary, time-sensitive procedures" (MeNTS) scoring system, urology consensus recommendations, and individual surgeon case prioritization for resumption of elective urologic surgery during the COVID-19 Pandemic [published online ahead of print, 2020 Jul 27]. J Urol. 2020;205(1):241-247. doi:10.1097/ JU.0000000000001315

52. Wallis CJD, Catto JWF, Finelli A, et al. The Impact of the COVID-19 pandemic on genitourinary cancer care: re-envisioning the future. Eur Urol. 2020;78(5):731-742. doi:10.1016/j.eururo.2020.08.030
Risk Management and Healthcare Policy

\section{Publish your work in this journal}

Risk Management and Healthcare Policy is an international, peerreviewed, open access journal focusing on all aspects of public health, policy, and preventative measures to promote good health and improve morbidity and mortality in the population. The journal welcomes submitted papers covering original research, basic science, clinical \& epidemiological studies, reviews and evaluations, guidelines, expert opinion and commentary, case reports and extended reports. The manuscript management system is completely online and includes a very quick and fair peer-review system, which is all easy to use. Visit http://www.dovepress.com/testimonials.php to read real quotes from published authors. 